\title{
Genetic and environmental factors drive personalized medicine for Crohn's disease
}

\author{
Shigeru Oshima and Mamoru Watanabe \\ Department of Gastroenterology and Hepatology, Graduate School, Tokyo Medical and Dental University (TMDU), Tokyo, Japan.
}

\begin{abstract}
The introduction of anti-TNF antibody therapy has changed the course of treatment for Crohn's disease. However, the fundamental mechanism for the onset of Crohn's disease is still unknown, and the treatment strategy for this disease remains suboptimal. The assessment of the disease phenotype based on key environmental factors and genetic background may indicate options for the personalized treatment of Crohn's disease. In this issue of the $J C I$, Liu et al. show that consumption of tobacco and the mutation of ATC16 ${ }^{\text {T300A }}$, a prevalent Crohn's disease susceptibility allele, drive defects in cells at the bottom of the intestinal crypt, the Paneth cells. These factors may provide novel targets for personalized medicine.
\end{abstract}

a prevalent Crohn's disease susceptibility allele, introduces a caspase-cleavage site that destabilizes the protein product and reduces autophagy in the presence of TNF- $\alpha$ $(7,8)$. Studies have demonstrated hypomorphic ATG16L1 protein expression, reduced autophagy, and abnormalities in Paneth cells in mutant mice (9). Importantly, patients with Crohn's disease who are homozygous for the ATG16L1 allele showed Paneth cell abnormalities similar to those observed in mice. Paneth cells are thought to be the primary site from which intestinal inflammation originates (10). An abnormal Paneth cell phenotype is associated with mucosal dysbiosis and an aggressive disease course $(11,12)$. Moreover, the Paneth cell phenotype may be a biologically and clinically relevant biomarker, able to stratify patients with Crohn's disease. However, the factors inducing the abnormality in Paneth cells are currently unknown. Currently, it is hypothesized that various environmental factors may be involved in this process.

\section{Link between the risk of Crohn's disease and environmental factors}

The key mechanism underlying the pathogenesis of Crohn's disease involves environmental factors triggering inflammation in genetically susceptible patients. There are several well-defined or putative environmental risk factors, including use of tobacco (13), dietary factors (14), and some kind of drug use (e.g., antibiotic and nonsteroidal antiinflammatory drugs) $(15,16)$. Recent studies have suggested potential interactions between genetic and environmental factors. An increased n-6/n-3 polyunsaturated fatty acid (PUFA) ratio is associated with an increased risk of Crohn's disease in children who are carriers of specific variants of the monooxygenases CYP4F3 and FADS2, genes involved in the regulation of PUFA metabolism (17). In established Crohn's disease, use of tobacco has been clinically associated with greater disease activity and early postop- 


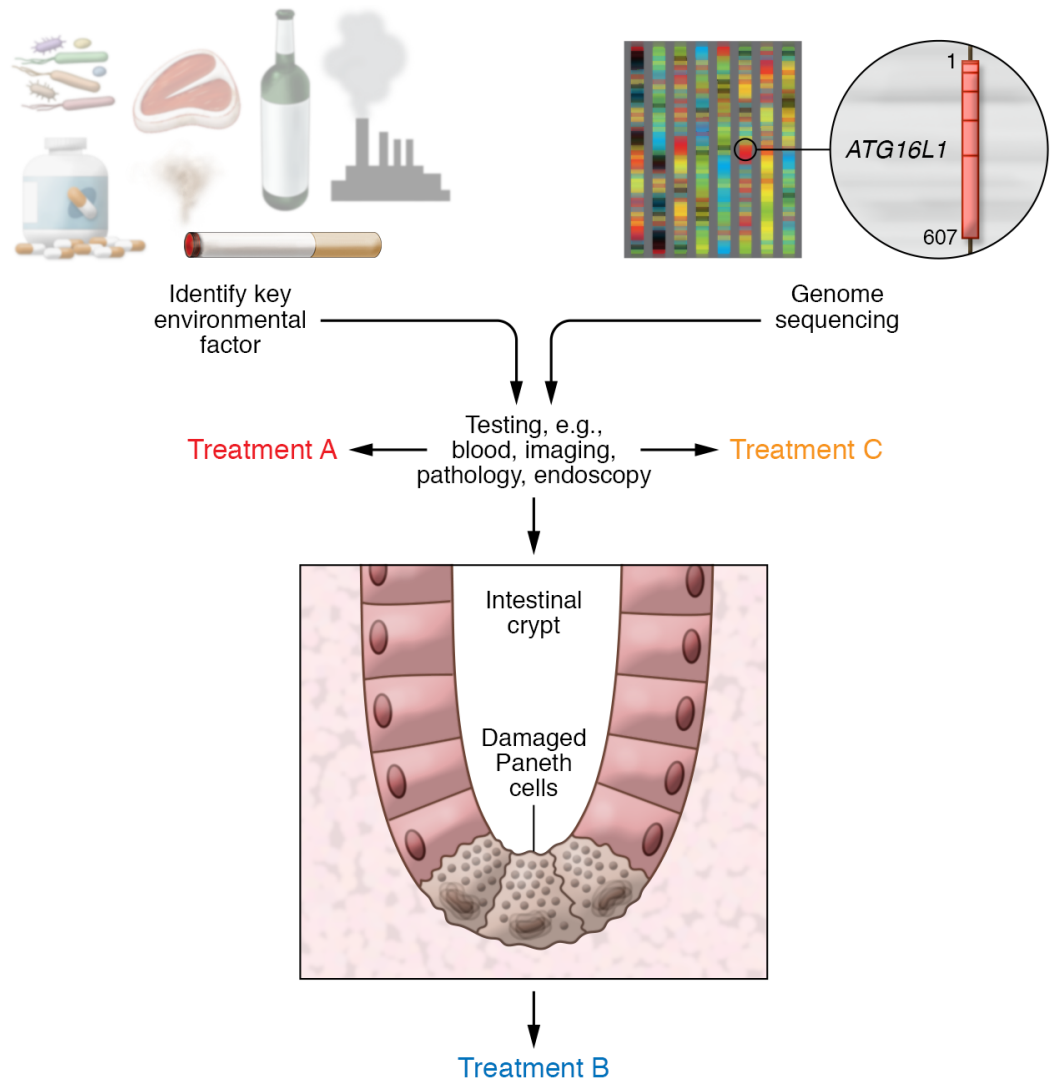

Figure 1. The approach of personalized medicine for Crohn's disease. After screening of environmental factors and the genetic background, confirmation of appropriate biomarkers determines therapeutic strategies.

erative recurrence. Studies have demonstrated a significant link between the risk of Crohn's disease and factors such as tobacco smoking, genetic variants in the cytochrome CYP2A6/Egl nine homolog 2 (EGLN 2) locus, and glutathione transferase enzymes (GSTP1) (18).

\section{Targeting Paneth cells in Crohn's disease}

In this issue, Liu et al. (19) show that the smoking status and Paneth cell phenotype may be prognosticators of recurrence in patients with Crohn's disease who received ileocolonic anastomosis and postoperative immunomodulatory and/or biologic prophylactic therapy. The combination of these factors further stratified patients into prognostically distinct subgroups. In addition, patients with Crohn's disease carrying the $A T G 16 L 1^{T 300 A}$ genotype who were also smokers (T300A-smoking group) showed a significantly shorter time to recurrence after surgery. Recent studies have suggested that necroptosis modulates Paneth cell function $(20,21)$. How- ever, smoking-induced Paneth cell defects in T300A mice were rescued only by inhibition of apoptosis, but not through inhibition of necroptosis. Liu et al. show that apoptosis was a central mechanism behind smoking-associated Paneth cell defects in patients with Crohn's disease and mice with the $A T G 16 L 1^{T 300 A}$ genotype. Importantly, apoptosis and Paneth cell defects were regulated by PPAR $\gamma$ signaling, which may be a potential target for treatment.

Personalized medicine involves the determination of patients at high risk of disease progression and occurrence of complications. Moreover, it includes the characterization of patients who may respond preferentially to specific therapies (Figure 1). In addition to drug discovery based on personalized medicine, the comprehensive exploration of biomarkers is necessary for the optimization of treatment using currently available therapeutic agents. The development of personalized medicine for patients with Crohn's disease is not only possible, but is an attractive prospect. The identification of biomarkers able to predict the efficacy of molecular-targeted drugs prior to their administration reduces time to remission and increases the efficacy of the drugs. In addition, this process may contribute greatly to the medical economy. Recent studies have been aimed at identifying factors predictive of drug response (corresponding to a specific signaling pathway) for the improved management of patients with Crohn's disease. Furthermore, imaging techniques applicable in personalized medicine have advanced considerably in recent years. Ultrasonography, computed tomography, and magnetic resonance enterography $(22,23)$ are widely used for the evaluation of inflammation in the gut. Prognostic and therapeutic subgroups in Crohn's disease are determined based on clinical characteristics and genetic-environmental factor interactions for the selection of the most appropriate treatment. Liu et al. investigated the link between a genetic susceptibility allele $\left(A T G 16 L 1^{T 300 A}\right)$ and an environmental factor (smoking) and the development of a morphological change (Paneth cell defects) that ultimately results in a distinct phenotype. The investigation of environmental and genetic factors, coupled with the evaluation of morphological changes through endoscopy and/or pathological biopsy, permits the use of personalized medicine for the treatment of Crohn's disease.

\section{Concluding remarks}

In conclusion, Crohn's disease is considered the result of interplay between genetic susceptibility and environmental factors. Based on the relationship between the mutation on the susceptibility gene and the smoking status of patients, the Paneth cell phenotype may define the therapeutic effect. In the future, the selection of therapeutic strategies may be determined through the combination of various environmental factors and genetic mutations. The use of personalized medicine based on this approach may provide more effective and safer therapies for Crohn's disease.

Address correspondence to: Mamoru Watanabe, Department of Gastroenterology and Hepatology, Graduate School, Tokyo Medical and Dental University (TMDU), 1-5-45, Yushima, Bunkyo-ku, Tokyo 1138519, Japan. Phone: 81.3.5803.5877; Email: mamoru.gast@tmd.ac.jp. 
1. Abraham C, Cho JH. Inflammatory bowel disease. N Engl J Med. 2009;361(21):2066-2078.

2. Torres J, Mehandru S, Colombel JF, Peyrin-Biroulet L. Crohn's disease. Lancet. 2017;389(10080):1741-1755.

3. Shah SC, Colombel JF, Sands BE, Narula N. Systematic review with meta-analysis: mucosal healing is associated with improved long-term outcomes in Crohn's disease. Aliment Pharmacol Ther. 2016;43(3):317-333.

4. McGovern DP, Kugathasan S, Cho JH. Genetics of inflammatory bowel diseases. Gastroenterology. 2015;149(5):1163-1176.e2

5. Jostins L, et al. Host-microbe interactions have shaped the genetic architecture of inflammatory bowel disease. Nature. 2012;491(7422):119-124.

6. Lee JC, et al. Genome-wide association study identifies distinct genetic contributions to prognosis and susceptibility in Crohn's disease. Nat Genet. 2017;49(2):262-268.

7. Lassen KG, et al. Atg16L1 T300A variant decreases selective autophagy resulting in altered cytokine signaling and decreased antibacterial defense. Proc Natl Acad Sci U S A. 2014;111(21):7741-7746.

8. Murthy A, et al. A Crohn's disease variant in Atg16l1 enhances its degradation by caspase 3 . Nature. 2014;506(7489):456-462.

9. Cadwell K, et al. A key role for autophagy and the autophagy gene Atg16l1 in mouse and human intestinal Paneth cells. Nature. 2008;456(7219):259-263.

10. Adolph TE, et al. Paneth cells as a site of origin for intestinal inflammation. Nature. 2013;503(7475):272-276.

11. VanDussen KL, et al. Genetic variants synthesize to produce paneth cell phenotypes that define subtypes of Crohn's disease. Gastroenterology. 2014;146(1):200-209.

12. Liu TC, et al. Paneth cell defects in Crohn's disease patients promote dysbiosis. JCI Insight. 2016;1(8):e86907.

13. Cosnes J, Carbonnel F, Beaugerie L, Le Quintrec Y, Gendre JP. Effects of cigarette smoking on the long-term course of Crohn's disease. Gastroenterology. 1996;110(2):424-431.

14. Ananthakrishnan AN, et al. A prospective study of long-term intake of dietary fiber and risk of Crohn's disease and ulcerative colitis. Gastroenterology. 2013;145(5):970-977.

15. Card T, Logan RF, Rodrigues LC, Wheeler JG. Antibiotic use and the development of Crohn's disease. Gut. 2004;53(2):246-250.

16. Ananthakrishnan AN, et al. Aspirin, nonsteroidal anti-inflammatory drug use, and risk for Crohn disease and ulcerative colitis: a cohort study. Ann Intern Med. 2012;156(5):350-359.
17. Costea I, et al. Interactions between the dietary polyunsaturated fatty acid ratio and genetic factors determine susceptibility to pediatric Crohn's disease. Gastroenterology. 2014;146(4):929-931.

18. Ananthakrishnan AN, Nguyen DD, Sauk J, Yajnik V, Xavier RJ. Genetic polymorphisms in metabolizing enzymes modifying the association between smoking and inflammatory bowel diseases. Inflamm Bowel Dis. 2014;20(5):783-789.

19. Liu TC, et al. Interaction between smoking and ATG16L1 $1^{T 300 A}$ triggers Paneth cell defects in Crohn's disease. J Clin Invest. 2018;128(11):5110-5122.

20. Günther C, et al. Caspase- 8 regulates TNF- $\alpha-$ induced epithelial necroptosis and terminal ileitis. Nature. 2011;477(7364):335-339.

21. Matsuzawa-Ishimoto Y, et al. Autophagy protein ATG16L1 prevents necroptosis in the intestinal epithelium. J Exp Med. 2017;214(12):3687-3705.

22. Takenaka K, et al. Comparison of magnetic resonance and balloon enteroscopic examination of the small intestine in patients with Crohn's disease. Gastroenterology. 2014;147(2):334-342.e3.

23. Panes J, Jairath V, Levesque BG. Advances in use of endoscopy, radiology, and biomarkers to monitor inflammatory bowel diseases. Gastroenterology. 2017;152(2):362-373.e3. 